\title{
Simultaneous and Complete Control of Light Polarization and Phase using High Contrast Transmitarrays
}

\author{
Amir Arbabi ${ }^{1}$, Yu Horie ${ }^{1}$, Mahmood Bagheri ${ }^{2}$, Andrei Faraon ${ }^{1}$ \\ ${ }^{I}$ T. J. Watson Laboratory of Applied Physics, California Institute of Technology, 1200 E California Blvd., Pasadena, CA 91125 \\ ${ }^{2}$ Jet Propulsion Laboratory, California Institute of Technology, Pasadena, CA 91109, USA \\ amir@caltech.edu
}

\begin{abstract}
We report an efficient dielectric metasurface platform for complete control over polarization and phase of light with subwavelength spatial resolution. Using this platform, we experimentally demonstrate polarization switchable phase holograms and vector beam generators.

OCIS codes: (050.6624) Subwavelength structures; (230.5440) Polarization-selective devices; (050.1965) Diffractive lenses.
\end{abstract}

Planar diffractive optical devices which can be mass produced at low cost using standard silicon microfabrication techniques have recently attracted significant attention [1]. Several different platforms such as plasmonic resonator arrays [1], high contrast gratings [2], and high contrast arrays [3-5] have been examined, and components such as lenses, mirrors, and wave plates are demonstrated. Among these platforms, the high contrast arrays have been shown to provide the largest efficiencies and the highest flexibility in the design of different components. High contrast arrays are composed of an array of potentially dissimilar weakly coupled elements with large refractive index which are positioned at the lattice sites of a periodic lattice, and are surrounded by materials of lower refractive index. Each of the elements scatters the light incident on it almost independently of its neighbors. The scattered light might have a different phase or polarization from the incident light; therefore, the phase or the polarization of the light can be significantly modified using such an array. High contrast arrays use lattices with subwavelength lattice constants which leads to high diffraction efficiencies since the nonzero diffraction orders are suppressed, and also provides high resolution sampling of the desired phase or polarization profiles. Microlenses based on the high contrast arrays with efficiencies in excess of $85 \%$ have been recently demonstrated [4]. Here, we present a platform based on high contrast arrays which provides simultaneous and complete control over the polarization and phase of the transmitted light with high efficiency and subwavelength spatial resolution. Using the proposed platform, we demonstrate two categories of diffractive optical devices: polarization switchable phase holograms and arbitrary vector beam generators. The devices from the first category impose two different phase profiles onto optical waves with two different polarizations, and devices from the second category generate desired polarization and phase distributions from an incident beam with a given polarization state.

Figure 1 (a) shows a schematic illustration of the high contrast array used to achieve full control over the phase of the transmitted light with two orthogonal linear polarizations. For the operating wavelength of $915 \mathrm{~nm}$, we use 715 $\mathrm{nm}$ tall elliptical amorphous silicon $(\mathrm{aSi})$ posts which rest on a fused silica substrate and are arranged in a hexagonal lattice with lattice constant of $650 \mathrm{~nm}$. The posts exhibit an effective birefringence and impose different phase shifts on the optical waves polarized linearly along the two ellipse axes. As the simulation results presented in Fig. 1 (b), and (c) show, by appropriate selection of the two diameters of elliptical posts, any combination of phase shifts $\phi_{x}$ and $\phi_{y}$ can be achieved while the average transmission remains high. To design a device that imposes two distinct phase profiles for the $x$ and $y$ polarized lights, we sample the phase profiles at the lattice sites and place elliptical posts with major and minor diameters corresponding to the sampled phases at the sampling locations. Using this method, we designed a polarization beam splitter/focuser which deflects the $x$ and $y$ polarized beams by $+5^{\circ}$ and $-5^{\circ}$ degrees, respectively, and focuses them into two different points $1 \mathrm{~mm}$ away from the device (as shown schematically in Fig. 1 (d)). To design a device that generates a desired polarization and phase distribution from a given incident beam, we first sample the given input and the desired output field at the lattice sites, and compute a unitary and symmetric Jones matrices that map the sampled input fields to the sampled output fields at each site. Then, we implement the computed Jones matrices using elliptical posts with different diameters and orientations located at these sites. The details of this procedure are presented in [5]. Using this method, we designed a device that converts $x$ and $y$ polarized beams to radially and azimuthally polarized beams, respectively, and focuses them to doughnut shaped spots $200 \mu \mathrm{m}$ away from the device. A schematic illustration of this device is shown in Fig. 1 (e).

The devices were fabricated on a fused silica substrate. We deposited $715 \mathrm{~nm}$ aSi using plasma enhanced chemical vapor deposition (PECVD). Next, we spun positive ebeam resist and patterned it using electron beam lithography. 
(a)

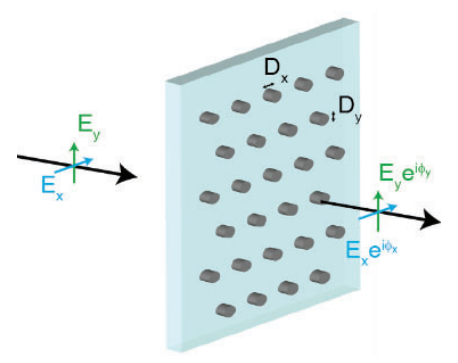

(d)

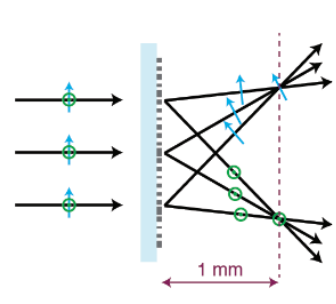

(e)

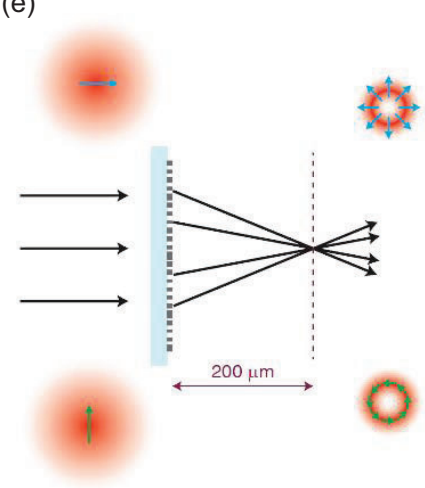

(b)

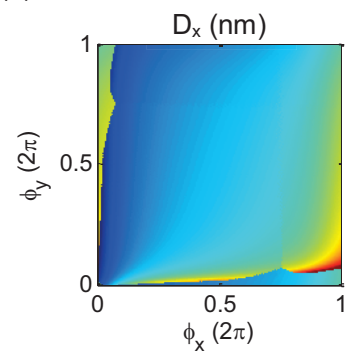

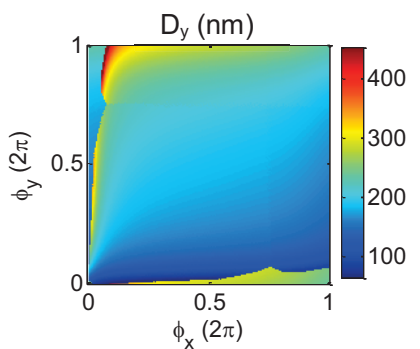

(c)

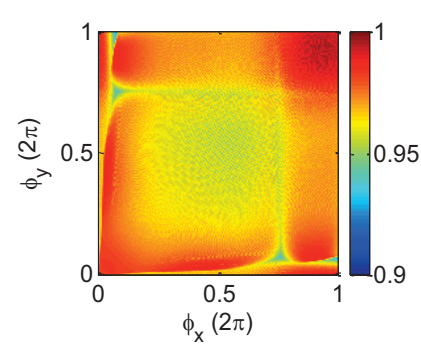

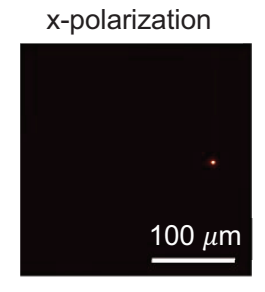
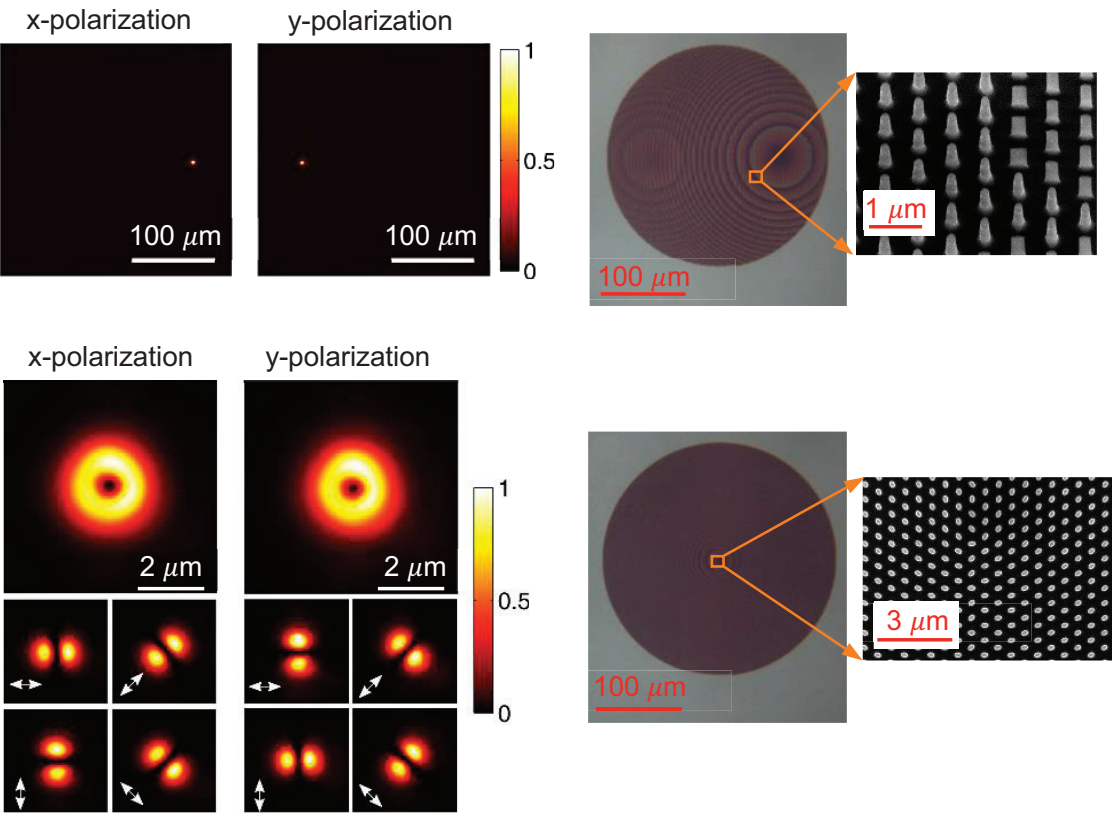

Fig. 1 (a) An array of elliptical aSi posts that exhibits effective birefringence. (b) Color coded values of the diameters of the elliptical posts along $x$, and $y$ directions for achieving desired phase shifts $\phi_{x}$ and $\phi_{y}$ for the $x$ and $y$ polarizations. (c) Color coded values of the average transmission of the array shown in (a) with corresponding diameters shown in (b). (d) Schematic illustration, measurement results, and optical and scanning electron microscope images for a polarization beam splitter/focuser, and (e) for a radial/azimuthal beam convertor and focuser.

A $70 \mathrm{~nm}$ thick aluminum oxide layer was then deposited on the developed resist, and was patterned by lifting up the resist. The patterned aluminum oxide was used as a hard mask for dry etching of aSi, and was subsequently removed using a mixture of ammonium hydroxide and hydrogen peroxide.

We characterized the devices by illuminating them with $915 \mathrm{~nm}$ polarized light, and imaging the diffracted light's intensity at the planes shown by dashed lines in the schematics shown in Figs. 1(d) and (e). The measurement results are shown in Figs. 1 (d) and (e). Projected intensities along different polarization directions (which confirm the radial and azimuthal polarization of the output beam) are also shown in Fig. 1 (e). Optical and electron microscope images of the fabricated devices are also presented. The dielectric metasurface design reported here provides a unified platform for fabrication of conventional and novel free-space optical components using a single lithography step. These components can operate as discrete elements or be seamlessly integrated with photonic circuits, fibers, and optoelectronic systems.

\section{References}

[1] N. Yu and F. Capasso, "Flat optics with designer metasurfaces" Nature materials 13, 139 (2014).

[2] D. Fattal, J. Li, Z. Peng, M. Fiorentino, and R. G. Beausoleil, "Flat dielectric grating reflectors with focusing abilities," Nature Photonics 4, $466(2010)$.

[3] S. Vo, et al. "Sub-wavelength grating lenses with a twist," IEEE Photonics Technology Letters 26, 1 (2014).

[4] A. Arbabi, M. Bagheri, A. J. Ball, Y. Horie, and A. Faraon, "Subwavelength-thick lenses with high numerical apertures and large efficiency based on high contrast transmitarrays,"Arxiv, arxiv:1410.8261.

[5] A. Arbabi, M. Bagheri, Y. Horie, and A. Faraon, "Complete control of polarization and phase of light with high efficiency and subwavelength spatial resolution,” Arxiv, arxiv:1411.1494. 